\title{
The performance of the SCRIT detectors for electron-RI scattering experiment
}

\author{
A. Enokizono*', K. Adachi, T. Fujita, M. Hori, K. Kurita, S. Sasamura, M. Togasaki, N. \\ Uchida, K. Yamada \\ Department of Physics, Rikkyo University, Toshima-ku, Tokyo 171-8501, Japan
}

K. Kasama, K. Namba, T. Suda, T. Tamae, K. Tsukada

Research Center for Electron-Photon Science, Tohoku University, Sendai, Miyagi 981-0928,

Japan

M. Hara, T. Hori, S. Ichikawa, T. Ohnishi, M. Wakasugi, M. Watanabe

Nishina Center, RIKEN, Wako, Saitama 351-0198, Japan

\section{S. Wang}

School of space science and physics, Shangdong University

\begin{abstract}
The SCRIT (Self-Confining RI Ion Target) facility has been constructed at RIKEN to make the electron-RI scattering experiment possible for the first time in the world. At SCRIT, the angular momentum distribution of scattered electrons is measured by WiSES (Window-frame Spectrometer for Electron Scattering) which consists of a dipole magnet and front/rear drift chambers, covering a solid angle of $\sim 80 \mathrm{mSr}$ with an intrinsic momentum resolution of $\Delta p / p \sim 10^{-3}$. Also the luminosity of the electron-RI scattering is estimated from bremsstrahlung photons measured by LMon (Luminosity Monitor) to obtain the absolute cross-section of the scattering. The LMon consists of a CsI calorimeter to measure the photon energy, and plastic fiber scintillators to measure the 2D hit distribution of the bremsstrahlung photons. In 2015-2016 the first physics experiment has been performed using ${ }^{132} \mathrm{Xe}$ target, then the luminosity and angular distributions were successfully measured at the electron beam energy of $150-300 \mathrm{MeV}$, achieving a luminosity above $10^{27} \mathrm{~cm}^{-2} \mathrm{~s}^{-1}$. In this article, we present the performances of the WiSES and LMon detectors obtained by using the ${ }^{132} \mathrm{Xe}$ experiment data.
\end{abstract}

The 26th International Nuclear Physics Conference

11-16 September, 2016

Adelaide, Australia

\footnotetext{
*Speaker.

†enoki@rikkyo.ac.jp
} 


\section{Introduction}

Electron scattering became one of the most powerful tools to measure the detailed internal structure of nucleus since the method was established by R. Hofstadter [1]. In the method, the angular differential cross-section of elastically scattered electrons is expressed by the Plane-Wave Impulse Approximation (PWIA) as

$$
\left(\frac{d \sigma}{d \Omega}\right)=\left(\frac{d \sigma}{d \Omega}\right)_{\text {mott }}\left|F_{c}(q)\right|^{2},
$$

where $(d \sigma / d \Omega)_{\text {mott }}$ is the angular differential of Mott scattering and $F_{c}(q)$ is the charge form factor with the momentum transfer $q$. Although the charge density distributions have been successfully measured for many stable nuclei by former electron scattering experiments, the technique has never been applied for short-lived radioactive isotope (RI) since it was technically difficult to achieve a high luminosity of electron scattering with short-lived targets. In order to make the experiment of electron scattering off short-lived nuclei possible, the SCRIT (Self-Confining RI ion Target) device has been studied and developed at RIKEN [2, 3]. The construction of the SCRIT facility has started at RIKEN RIBF building when the electron storage ring, named "SCRIT-equipped Riken Storage Ring" (SR2) was inherited from Sumitomo Heavy Industries in 2009. The schematic view of the SCRIT facility is shown in Fig 1. The electron beam provided by Race-Track Microtron (RTM) is transported to SR2 at the beam energy $E_{e}=150 \mathrm{MeV}$, then SR2 is capable of accelerating the beam up to $300 \mathrm{MeV}$. A chamber which contains the SCRIT device is installed at one of the straight lines of SR2. A detailed information and a recent progress on the SCRIT device is reported elsewhere in this proceedings [4].

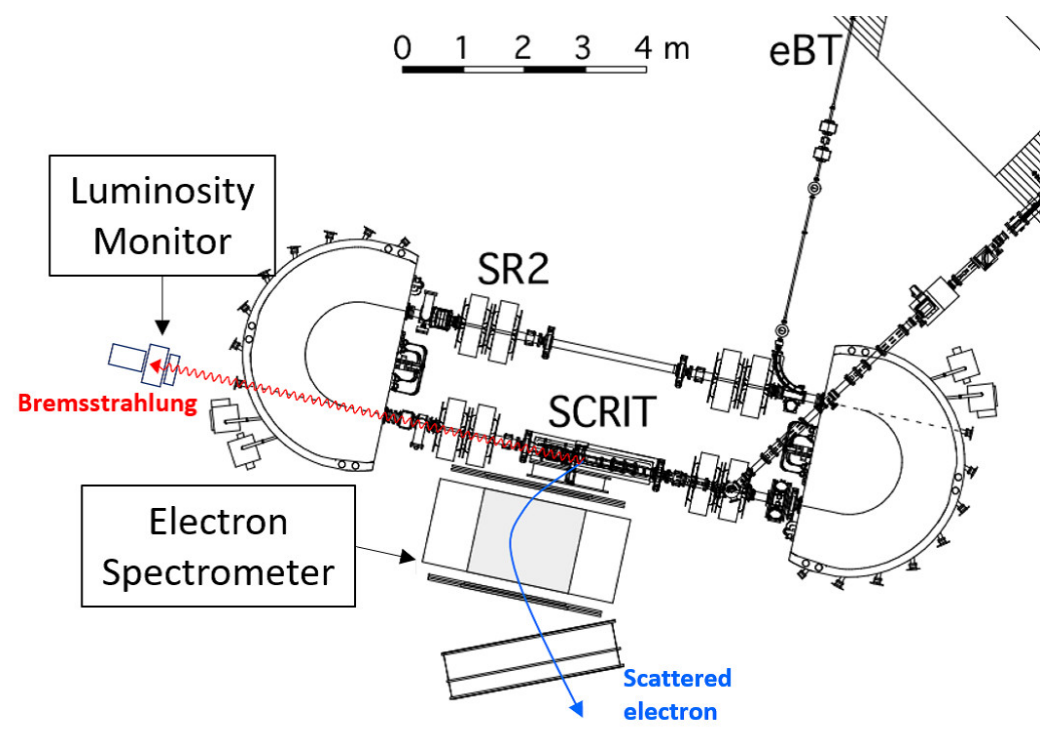

Figure 1: A schematic view of the SCRIT facility as of 2016.

To obtain the angular distribution of electron-nucleus scattering cross-section while its crosssection is still unknown, the absolute luminosity of electron-nucleus scatterings has to be measured 
in addition to the angular momentum distribution of the elastic scattering as

$$
\left(\frac{d \sigma}{d \Omega}\right)=\frac{1}{L}\left(\frac{d N}{d \Omega}\right)
$$

A SCRIT electron spectrometer, called Window-frame Spectrometer for Electron Scattering (WiSES), has been installed to measure the angle and momentum distributions of scattered electrons $(d N / d \Omega)$, and a luminosity monitor (LMon) was also installed to determine the absolute luminosity of the electron scattering $(L)$ by measuring bremsstrahlung photons.

In 2015-2016, the first physics experiment using stable ${ }^{132} \mathrm{Xe}$ target has been performed at $\mathrm{E}_{\mathrm{e}}=150-300 \mathrm{MeV}$. A typical maximum beam current when the data acquisition started is $\sim 280 \mathrm{~mA}$ and the beam life time is $\sim 40-70 \mathrm{~min}$. In order to estimate the background from residual gases inside the SR2 and SCRIT device, WiSES and LMon data have to be taken for both periods with and without ${ }^{132} \mathrm{Xe}$ ions trapped (Ion IN and Ion OUT), then net values of electron scatterings from target ${ }^{132} \mathrm{Xe}$ are derived from Ion IN-OUT. The trapping periods of Ion IN and Ion OUT occur alternately with a gap of $10 \mathrm{msec}$, and the each trapping time is equally set to 240 msec to maximize the luminosity or $990 \mathrm{msec}$ for a study of trapping phenomena using LMon, as described later. The physics result from the ${ }^{132} \mathrm{Xe}$ experiment is reported elsewhere in this proceedings [5], and the performances of the SCRIT detectors that were measured using the experiment data are described in the following sections.

\section{WiSES}

The WiSES was designed to measure scattered electrons, which are emitted from the SCRIT interaction region of $40 \mathrm{~cm}$, for a wide acceptance $\left(\theta \sim 30^{\circ}-60^{\circ}, \Omega \sim 80 \mathrm{mSr}\right)$ with a good momentum resolution $\left(\Delta p / p \sim 10^{-3}\right)$. It mainly consists of the front and rear drift chamber (FDC and RDC) with a dipole magnet in between as shown in Fig 2 The FDC has a volume of $117 \mathrm{~cm}(\mathrm{~W})$ $\times 25 \mathrm{~cm}(\mathrm{H}) \times 20 \mathrm{~cm}(\mathrm{D})$ and is made of 128 sense wires that form four layers as XX' XX', and the $\mathrm{RDC}$ has a volume of $274 \mathrm{~cm}(\mathrm{~W}) \times 36 \mathrm{~cm}(\mathrm{H}) \times 78 \mathrm{~cm}(\mathrm{D})$ and is made of 1002 sense wires that form 10 layers as UU'VV'XX'UU'VV'. U and V wires are tilted 45 and -45 degrees from $X$ wires respectively to acquire the 3 -dimensional position sensitivity. Both drift chambers are operated with $\mathrm{He}+\mathrm{C}_{2} \mathrm{H}_{6}(80: 20)$ gas. The 55 tons weighted dipole magnet has a window gap of $170 \mathrm{~cm}(\mathrm{~W}) \times$ $24 \mathrm{~cm}(\mathrm{H}) \times 140 \mathrm{~cm}(\mathrm{D})$ and is capable of applying a uniform vertical magnetic field at a maximum of $\sim 0.8 \mathrm{~T}$ to bend scattered electrons up to $300 \mathrm{MeV}$. The field clamps placed at the both sides of the gap ensure that the leak of the magnetic field is limited less than $0.1 \%$. The straight line tracking of passing electrons is performed at each drift chamber, then the trajectory in the dipole magnet is reconstructed using a 3-D map of OPERA-3D calculation. A helium filled bag made of $30 \mu$ thick vinyl sheet was inserted in the path of scattered electrons to minimize the multiple scattering effect. A pair of $2 \mathrm{~m}$ long plastic scintillators have been installed after the RDC as a event trigger hodoscope. Also as shown in Fig2 some other plastic scintillators were also installed in order to veto background events, i.e shower backgrounds originate from the SR2 beam, showers generated by electrons that hit the inside wall of the dipole magnet, and cosmic events.

Fig 3 shows the reconstructed momentum distributions of scattered electron for Ion IN and Ion OUT periods at $E_{e}=200 \mathrm{MeV}$. The Ion IN distribution apparently exceeds that of Ion OUT, and 


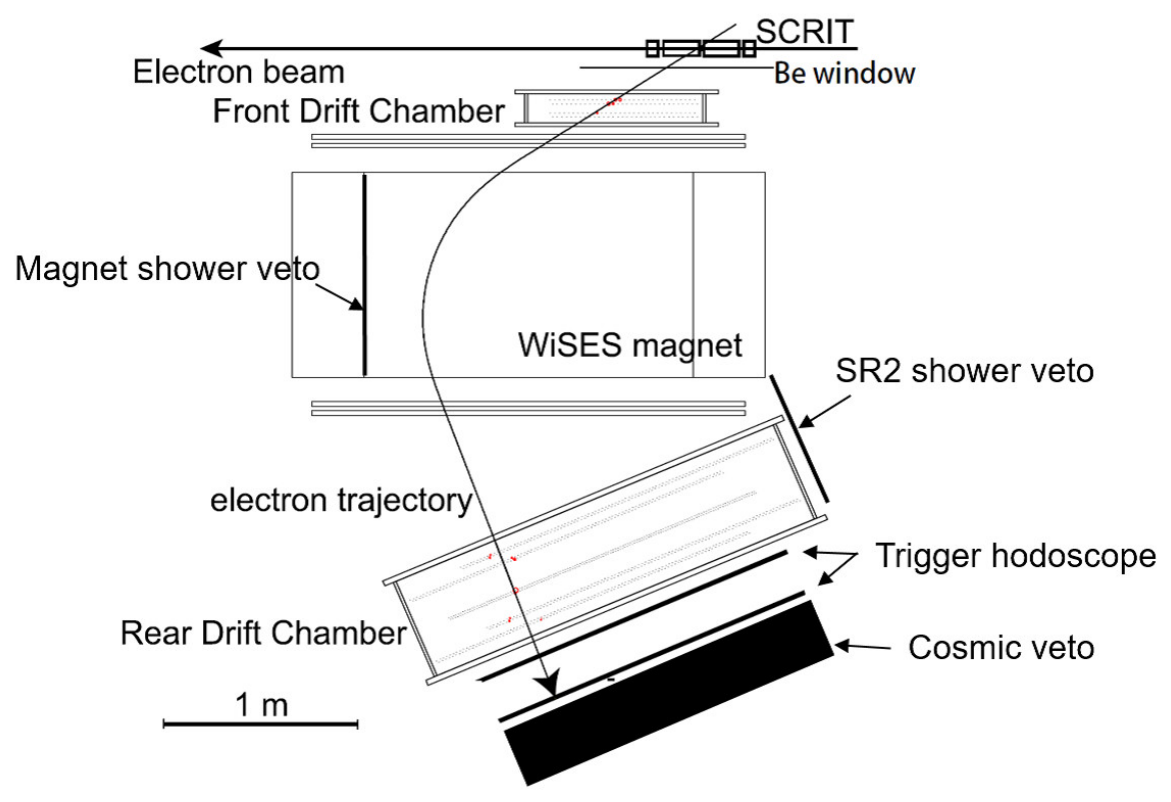

Figure 2: A diagram of WiSES apparatus and tracking.

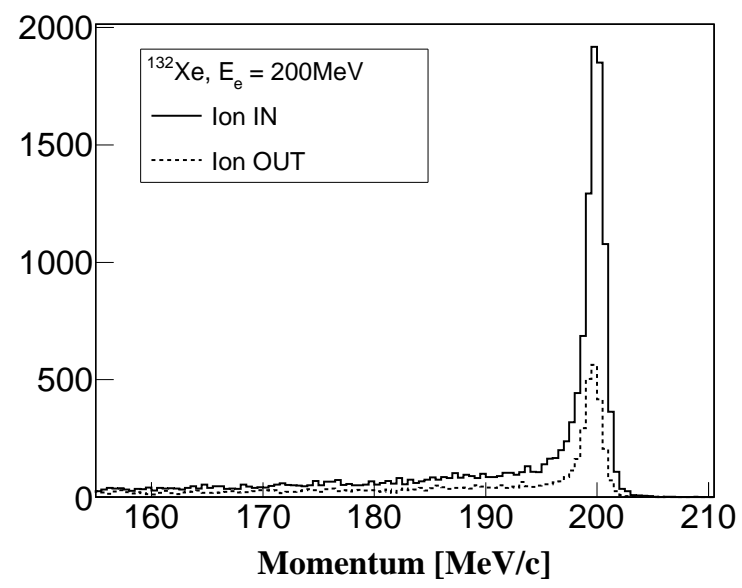

Figure 3: Momentum distributions of scattered electrons for Ion IN (solid line) and Ion OUT (dashed line).

the difference (Ion IN-OUT) is accounted as the momentum distribution of electrons scattered from ${ }^{132} \mathrm{Xe}$ target. The left panels of Fig 4 show the momentum distributions of Ion IN-OUT at $\mathrm{E}_{\mathrm{e}}=150$ $300 \mathrm{MeV}$. By fitting the distributions with Gaussian, the energy dependence of the momentum resolution is obtained as shown in the right panel of Fig 4 Comparing with an intrinsic momentum resolution estimated by GEANT4 detector simulation, the momentum resolution measured by the data is still 50\% worse. The momentum resolution is still sufficient to distinguish between elastic and inelastic scatterings for the coming day-one experiment using ${ }^{132} \mathrm{Sn}$ which has the $2^{+}$excited state at $\sim 4 \mathrm{MeV}$, but may not be good enough for other RIs which have shallower excitation energies. More studies on the momentum resolution of WiSES are underway now. 

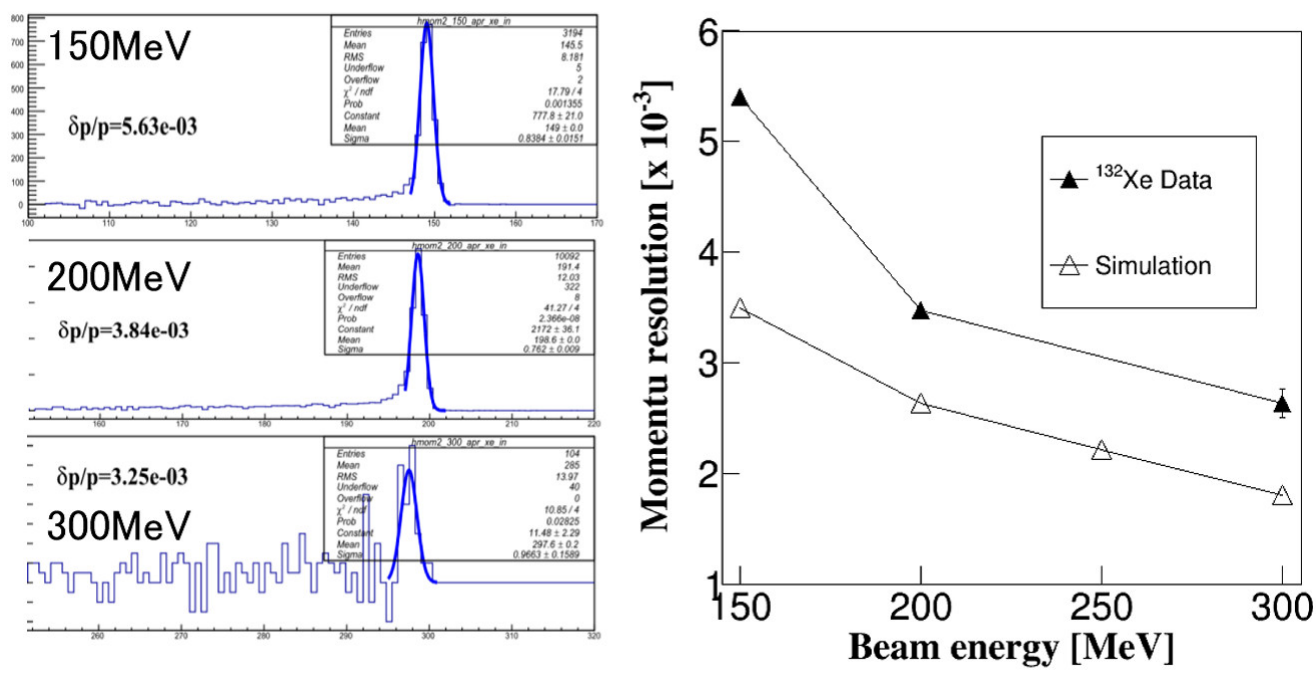

Figure 4: (Left panels) Momentum distributions of scattered electrons estimated from Ion IN-OUT at $\mathrm{E}_{\mathrm{e}}=150,200,300 \mathrm{MeV}$. (Right panel) Estimated momentum resolutions of WiSES as a function of $\mathrm{E}_{\mathrm{e}}$.

\section{LMon}

The absolute luminosity of electron-RI scattering can be estimated from the number of the bremsstrahlung photons since the cross-section of bremsstrahlung process is calculatable with a little ambiguity even for RIs which have not previously been measured. Here the luminosity can be obtained as

$$
L=\frac{N_{b r}}{t} \frac{1}{\varepsilon} \frac{1}{\sigma_{b r}}
$$

where $N_{b r} / t$ is the count rate of measured bremsstrahlung photons and $\sigma_{b r}$ is a calculated bremsstrahlung cross-section. $\varepsilon$ represents the detector acceptance for the bremsstrahlung photons including the detection efficiency. Since the photon detector cannot be placed inside the SCRIT-SR2, the best option to measure the bremsstrahlung photons is to put the LMon outside a SR2 view port which is $7 \mathrm{~m}$ downstream along the beam line from the interaction region as shown in Fig 1 The LMon has two main components; a CsI calorimeter to measure $N_{b r} / t$ and a fiber scintillator to measure $\varepsilon$, as illustrated in Fig 5. The CsI calorimeter is made out of 7 pure-CsI crystals and each crystal has a hexagonal shape with the side length of $4 \mathrm{~cm}$ and the depth is $20 \mathrm{~cm}$ long which is equivalent to $\sim 11$ radiation lengths. The energy calibration of each crystal was done by comparing cosmic data and GEANT4 simulation. The lead collimator is placed in front of the CsI calorimeter to reject shower backgrounds and assure that bremsstrahlung photons only hit the center CsI crystal, and surrounding 6 CsI crystals cover a small fraction of the total energy deposit, typically up to $20 \%$. The left panel of Fig 6 shows the total energy distribution measured by for the CsI calorimeter for ${ }^{132} \mathrm{Xe}$ target at $\mathrm{E}_{\mathrm{e}}=150 \mathrm{MeV}$. The GEANT4 simulation reproduces the data well and the end point of the energy tail is properly located at $150 \mathrm{MeV}$. The bremsstrahlung cross-section $\sigma_{b r}$ is estimated using a model calculation [6].

The fiber scintillator, which consists of $\mathrm{X}$ and $\mathrm{Y}$ position sensitive detectors, was designed 


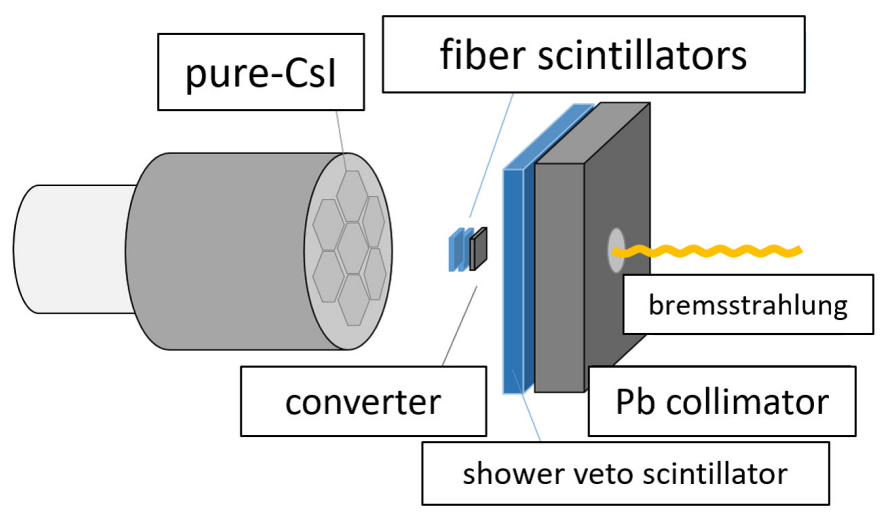

Figure 5: A schematic view of the LMon apparatus.

to measure the 2-dimensional hit position of the bremsstrahlung photons in order to estimate $\varepsilon$. Each position detector is made of stacked 16 plastic scintillators and each scintillator has a crosssection of $3 \mathrm{~mm} \times 3 \mathrm{~mm}$, covering the position sensitive area of $5 \mathrm{~cm} \times 5 \mathrm{~cm}$. To convert photons to electron-positron pairs, the aluminum converter is installed right before the fiber scintillator, and the conversion rate is about $20 \%$. The right panels of Fig 6 shows the hit distributions of the $\mathrm{X}$-Y fiber scintillators. The $\varepsilon$ can be estimated by using the GEANT4 simulation if the measured $\mathrm{X}-\mathrm{Y}$ hit distributions are well reproduced by those of GEANT4. However, there still is a small discrepancy in the hit distributions between the measured data and GEANT4, and it causes about $10 \%$ uncertainty of $\varepsilon$ which is the largest contribution to the total systematic uncertainty of $L$. Fig 7 shows the luminosity measured with the trapping time of $240 \mathrm{msec}$ as a function of the electron beam energy. It demonstrates that the luminosity increases as the beam energy increases, which is naturally explained by the fact that the beam size becomes bigger as the beam energy increases.

Detailed investigations into the ion trapping phenomenon by the SCRIT device were per-
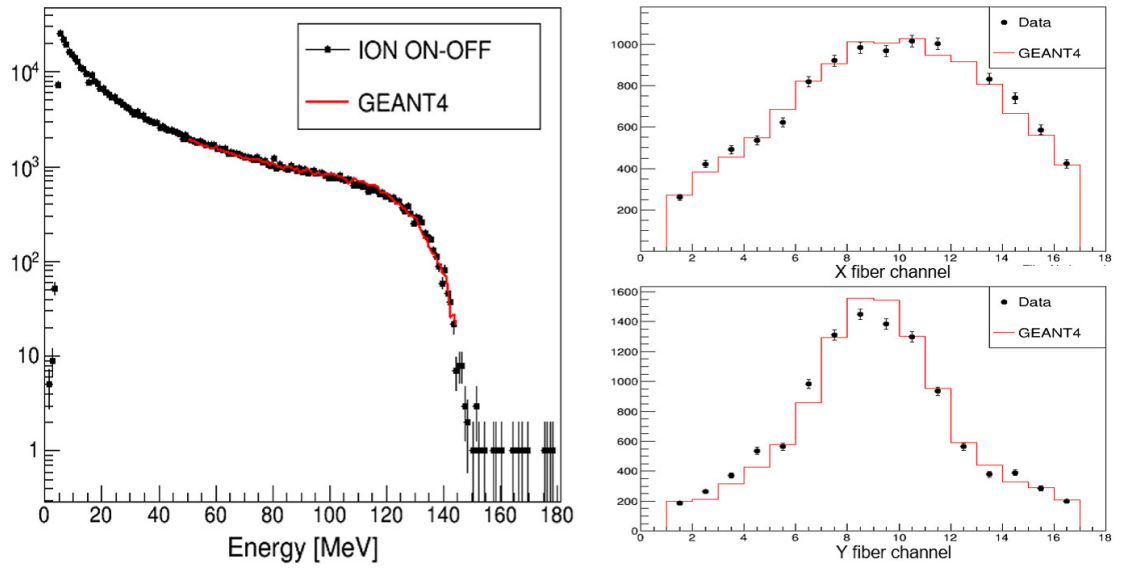

Figure 6: (Left panel) The total energy distribution of CsI at $\mathrm{E}_{\mathrm{e}}=150 \mathrm{MeV}$ compared with that of GEANT4 simulation. (Right panels) Hit distributions of X-Y fiber scintillators compared with GEANT4. 


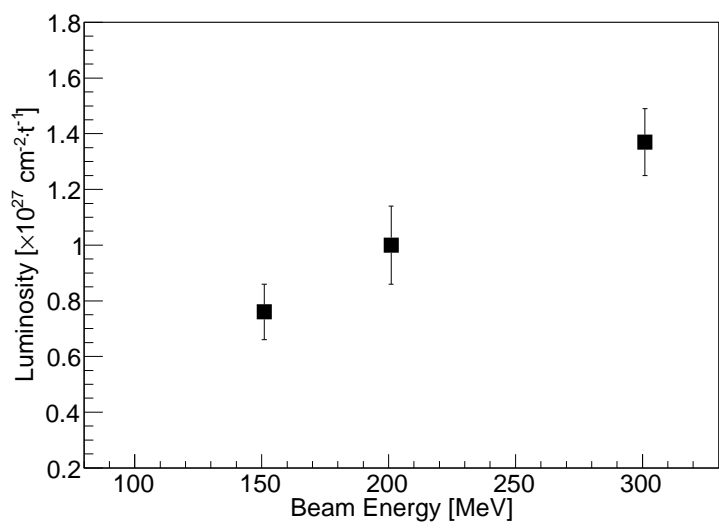

Figure 7: The measured luminosity of ${ }^{132} \mathrm{Xe}$ target as a function of the electron beam energy. The error bars indicate the total systematic uncertainty.

formed by using LMon. Fig 8 shows the photon counts by the CsI calorimeter as a function of the trapping time up to one second for different beam current regions using a ${ }^{132} \mathrm{Xe} 200 \mathrm{MeV}$ run. The photon counts from ${ }^{132} \mathrm{Xe}$ ions, which are shown by Ion IN-OUT histograms, indicate that the amount of the ion trapping decreases as the time of trapping elapses. On the other hand, the photon counts from the residual gas, which are shown by dashed lines (Ion OUT), indicate that the amount of the residual gas trapping actually increases as the function of the trapping time. It implies that the residual gas ions which are typically light nuclei like $\mathrm{H}_{2}, \mathrm{O}_{2}$ etc are trapped by the beam potential in a slower time scale than that of heavy target nuclei like ${ }^{132} \mathrm{Xe}$. Fig 9 shows the photon count rates of ${ }^{132} \mathrm{Xe}$ as a function of the trapping time for different current and beam energy regions. The count rate increases as the beam current and/or energy increases, which is caused by the bigger beam size as mentioned above. It appears that there are fast and slow components in the decay curves also for the target ${ }^{132} \mathrm{Xe}$, and the slow component becomes prominent as the beam

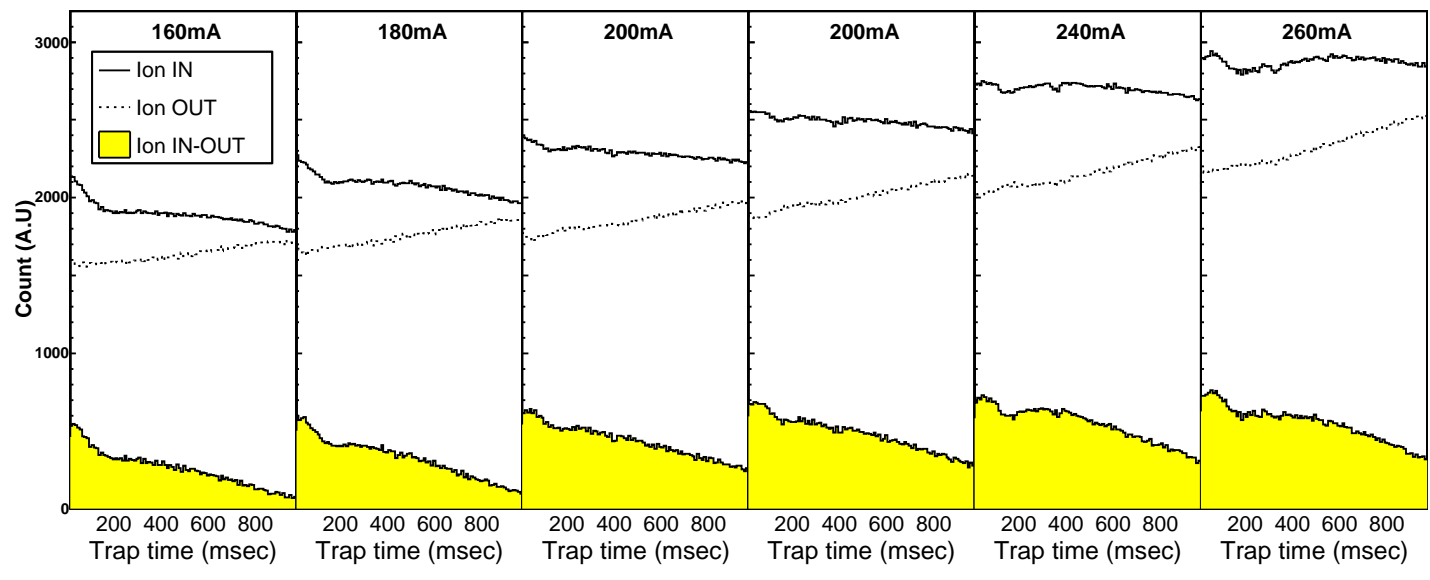

Figure 8: The trapping time dependences of CsI hit counts for Ion IN, Ion OUT and Ion IN-OUT measured for different current regions at $\mathrm{E}_{\mathrm{e}}=200 \mathrm{MeV}$. 

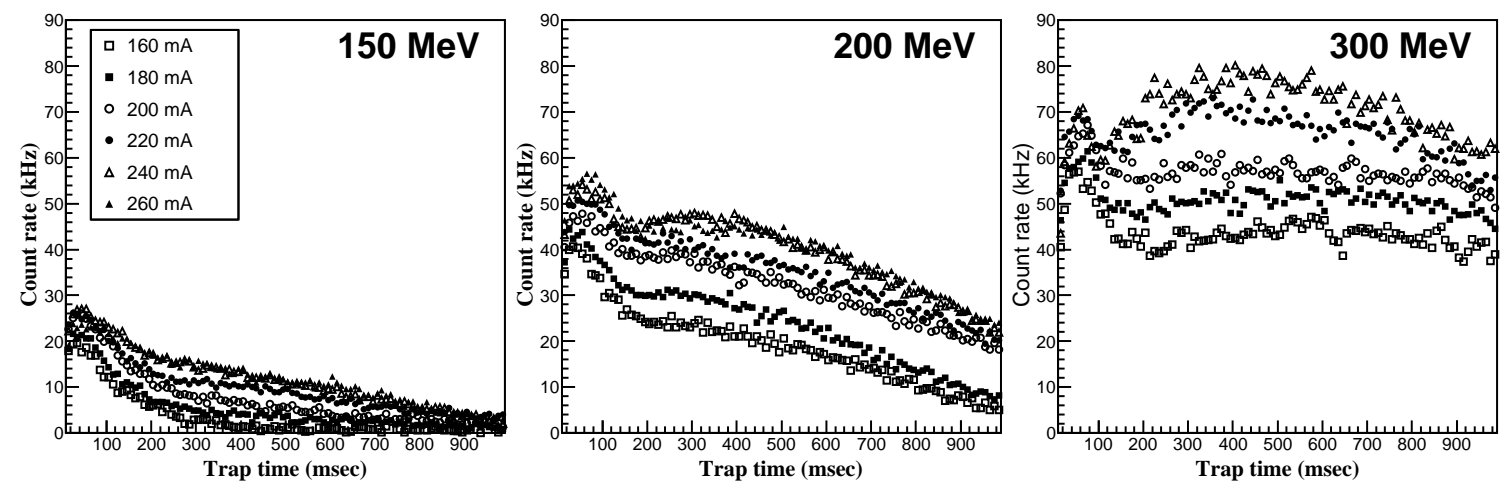

Figure 9: The trapping time dependence of the CsI hit rates for Ion IN-OUT measured for different current regions at $\mathrm{E}_{\mathrm{e}}=150 \mathrm{MeV}$ (left), $200 \mathrm{MeV}$ (center) and $300 \mathrm{MeV}$ (right).

energy and/or current increases. One possible explanation of the trend is that the number of highly charged state nuclei increases in the time scale of hundreds msec order. However, more detailed studies using simulations and calculations would be required to describe the phenomena for sure.

\section{Summary}

In summary, we have developed and installed the SCRIT detectors, LMon and WiSES, and the performances have been measured by the first physics experiment using ${ }^{132} \mathrm{Xe}$ target. As a result, the detector performances were found to be almost good as designed, and enough to perform the day-one experiment using ${ }^{132} \mathrm{Sn}$, but still there is room to be improved. The measured momentum resolution of WiSES is $\Delta p / p \sim 3 \times 10^{-3}$ at $\mathrm{E}_{\mathrm{e}}=300 \mathrm{MeV}$ which is still $\sim 50 \%$ worse than the intrinsic momentum resolution. Also the total systematic uncertainty of the measured luminosity by LMon is estimated to be more than $\sim 10 \%$ although it is expected to be better than the systematic uncertainty which originates from the angular resolution of WiSES $(\sim 5 \%)$. More investigations and studies using the GEANT4 simulation are underway to improve the performances, and the results will be reported in separate papers soon.

\section{References}

[1] R. Hofstadter, H. R. Fechter and J. A. McIntyre, High-Energy Electron Scattering and Nuclear Structure Determinations, Phys. Rev. 924 (1953) 978.

[2] M. Wakasugi et al, Novel Internal Target for Electron Scattering off Unstable Nuclei, Phys. Rev. Lett. 100 (2008) 164801.

[3] T. Suda et al, First Demonstration of Electron Scattering Using a Novel Target Developed for Short-Lived Nuclei, Phys. Rev. Lett. 102 (2009) 102501.

[4] T. Ohnishi et al, The Scrit Electron Scattering Facility At Riken RI Beam Factory, in this proceedings.

[5] K. Tsukada et al, First result from SCRIT electron scattering facility:Charge density distribution of ${ }^{132} \mathrm{Xe}$, in this proceedings.

[6] Y. S. Tsai, Pair production and bremsstrahlung of charged leptons, Rev. Mod. Phys. 46 (1974) 815. 